\title{
Transition processes for junction vortex flow
}

\author{
J. J. ALLEN ${ }^{1}$ AND J. M. LOPEZ \\ ${ }^{1}$ Department of Mechanical Engineering, New Mexico State University, Las Cruces, \\ NM 88003, USA \\ ${ }^{2}$ Department of Mathematics and Statistics, Arizona State University, Tempe, AZ 85287, USA
}

(Received 2 November 2006 and in revised form 16 April 2007)

The details of the start-up transient vortex structure that forms near the junction of an impulsively started plate and a stationary plate where a step jump in velocity occurs at the plate surfaces are investigated. Numerical simulations have been conducted in a geometry representative of recent experiments of this flow. The experiments did not have access to data at very early times following the impulsive start, but they did suggest that the flow undergoes transitions from a viscous-dominated phase to an inertia-dominated phase. The numerical simulations presented here are designed to explore the early viscous-dominated transients. The simulations show that when the non-dimensional time, $\tau=t U^{2} / v(t$ is the time that the plate has been in motion and $v$ is the kinematic viscosity), is less than 100, the development process is dominated by viscous forces. In this regime similarity scaling is used to collapse the data, which scale as $\sqrt{t}$. The simulation results at low $\tau$, when evaluated using entrainment diagrams, show an unsteady transition process consisting of the following stages. Initially, the flow consists of a non-rotating vorticity front with a single critical point for $\tau<40$. For $40<\tau<50$, the flow has three critical points, two nodes and a saddle. A rotational leading jet head develops for $\tau>50$ as the outermost node evolves into a spiral focus. The simulations span the viscous range to the inertial range. In the inertial range, for $\tau>10^{3}$, the flow structure scales as $t^{5 / 6}$, as was observed in the experiments.

\section{Introduction}

The study of flows that admit similarity analysis to reduce the system order has long fascinated researchers in fluid mechanics. It offers the potential to provide insight into complex unsteady flows. A class of flows that is amenable to similarity analysis is developing flows that are not constrained by an external or apparatus length scale. One such case is the flow in the region close to the junction of an impulsively started plate and a stationary plate where a step jump in velocity occurs at the plate surfaces, first considered by Taylor (1960). Experiments show that the region close to the junction is dominated by the evolution of a vortex roller if the wall speed is sufficiently high (Allen \& Naitoh 2007). If the length scales of an experimental or computational domain are large compared to the size of the structure that forms, there is the potential for a reduction in the order of the problem and a collapse of data in self-similar coordinates. The physical mechanism responsible for the formation of the vortex roller is that once the wall is set in motion, a vortex sheet forms over the moving wall. This thin vortex layer is driven over the stationary wall by the moving wall. A layer of vorticity of opposite sign and commensurate strength forms over the stationary wall to enforce the no-slip boundary condition. Consequently, the layer forming over the stationary wall resembles an unsteady wall jet started from rest. 
This dual-signed layer of vorticity then has the potential to separate and roll up into a vortex.

This type of transient structure has been observed when a piston moves through a cylinder (Guezet \& Kageyama 1997), as well as in wall-driven cavity flows (Koseff \& Street $1984 a, b)$. An outstanding question in the literature is, under what conditions does a vortex form? Hughes \& Gerrard (1971) estimated that for a Reynolds number of less than 450, where the length scale is based on piston diameter, no vortex formation is observed. Conlon \& Lichter (1995), in the study of the start-up of an unsteady wall jet, estimated that no rotational head was observed for a Reynolds number less than 50, based on the width of the wall jet. Both of these results imply that the apparatus length scale is relevant for the formation of a rotational structure. However, Tabaczynski, Hoult \& Keck (1970), Allen \& Chong (2000) and Allen \& Naitoh (2007) provide convincing evidence that the growth of the vortex, once formed, can be considered independent of the apparatus length scale. This immediately brings into question whether the notion of the formation of a vortex roller being dependent on an external length scale was appropriate in the earlier studies. Perhaps a more relevant question may be: How does the structure transition from a viscous one to an inertially dominated one?

In the study of a viscous jet generated from rest, Cantwell (1986) was able to show that the flow topology of an impulsively started jet is a function of the nature and size of the impulse imparted to the flow. At early non-dimensional time (i.e. low non-dimensional impulse) the starting flow resembles a moving, non-rotating, vorticity front. As the impulse is increased, the front develops a rotational head that is typically associated with a starting jet. Rotation in and of itself is not necessarily an important distinction; rather, it represented one of a series of bifurcations in the viscous flow topology. Analysis of our numerical results re-cast in similarity variables reveals an analogous development of the flow topology in the neighbourhood of the junction between the moving and stationary walls.

The goal of the current study is to utilize direct numerical simulations to establish the criteria for when a vortex roller forms. The numerics can readily span the parameter regime from viscous-dominated to inertia-dominated flow, thus complementing the experiments of Allen \& Naitoh (2007). Those experiments did not have access to reliable data in the early viscous regime.

\section{Governing equations and computational technique}

An idealized version of this problem consists of a flow in a semi-infinite domain, $x \in(-\infty, \infty), y \in[0, \infty), z \in(-\infty, \infty)$, driven by an impulsively started section of the lower boundary, $y=0$ and $x<0$, moving in the positive $x$-direction at constant speed $U$, with the boundary $y=0$ and $x>0$ remaining stationary. Of course, the physical experiment is conducted in a finite domain. For early times, the experimental flow remains essentially two-dimensional, i.e. invariant in the spanwise $z$-direction, except near the spanwise walls of the apparatus. Numerically, we shall take the flow as being $z$-independent, and solve the two-dimensional Navier-Stokes equations in a finite domain of depth $H$ and horizontal extent $2 H$. The moving part of the floor is $y=0$ and $x \in[-H, 0]$, the stationary part of the floor is $y=0$ and $x \in[0, H]$, and the junction is located at the origin, $(x, y)=(0,0)$.

The two-dimensional velocity is written in terms of a streamfunction, $\boldsymbol{u}=(u, v)=\left(\psi_{y},-\psi_{x}\right)$, where the subscripts denote partial differentiation. The only non-trivial component of vorticity is the spanwise component $\omega=-\psi_{x x}-\psi_{y y}$. In the 
streamfunction-vorticity formulation, the Navier-Stokes equations reduce to

$$
\omega_{t}+\psi_{y} \omega_{x}-\psi_{x} \omega_{y}=v\left(\omega_{x x}+\omega_{y y}\right) .
$$

The initial conditions are that everything is at rest at $t=0: \psi(x, y, 0)=$ $\omega(x, y, 0)=0$. The boundary conditions are no-slip. Specifically,

at the sidewalls:

on the top wall:

on the stationary part of the floor:

on the moving part of the floor:

$$
\begin{aligned}
\psi( \pm H, y, t) & =\psi_{x}( \pm H, y, t)=0 ; \\
\omega( \pm H, y, t) & =-\psi_{x x}( \pm H, y, t) ; \\
\psi(x, H, t) & =\psi_{y}(x, H, t)=0, \\
\omega(x, H, t) & =-\psi_{y y}(x, H, t) ; \\
\psi(x, 0, t) & =\psi_{y}(x, 0, t)=0, \\
\omega(x, 0, t) & =-\psi_{y y}(x, 0, t) ; \\
\psi(x, 0, t) & =\psi_{y}(x, 0, t)=U, \\
\omega(x, 0, t) & =-\psi_{y y}(x, 0, t)+\psi_{y}(x, 0, t) .
\end{aligned}
$$

We non-dimensionalize (2.1) and the boundary conditions using $H$ as the length scale, $U$ as the velocity scale and $H / U$ as the time scale, and define a Reynolds number

$$
R e=U H / \nu,
$$

where $v$ is the fluid's kinematic viscosity. The Reynolds number is the ratio of the inertial time scale $H / U$ and the viscous time scale $v / U^{2}$. Second-order finite differences are used to compute numerical solutions, using 1201 grid points in $x / H$ and 601 grid points in $y / H$ and a time step $\delta_{t} U / H$ which depends on $R e$. Further details on the numerical method and a discussion on the numerical treatment of jump discontinuities in boundary conditions are available in Lopez \& Shen (1998).

\section{Results}

Figure 1 shows vorticity contours in the full computational domain for $R e=10^{4}$ over a range of non-dimensional times $U t / H \in[1,10]$. Positive spanwise vorticity develops over the moving wall and negative spanwise vorticity develops over the stationary walls (including the top and end walls). Both these sheets of vorticity separate at the junction on the floor and roll up into the vortex structure. Localized self-similar behaviour of the vortex may be expected while the size of the developing vortex structure remains small compared to the computational domain, e.g. for $U t / H<4$ in the case shown in figure 1 . The separated vortex structure in figure $1(f)$, for example, is of the same size as the computational domain, and hence by this point one would expect that the presence of domain boundaries is having a significant effect on the flow scaling.

In analysing the results and testing for self-similarity, we have found it convenient to use the non-dimensional time $\tau=t U^{2} / \nu=\operatorname{Re} U t / H$. If, in the absence of experimental length scales, the flow field is universally defined by $\tau$, then simulations at various $R e$ should collapse at identical $\tau$ values.

Allen \& Naitoh (2007) showed that the velocity fields measured using particle image velocimetry (PIV) and flow visualizations appear to be similar at equivalent $\tau$ for values of $\tau>10^{3}$ (the smallest $\tau$ for which reliable data could be obtained) when space is scaled with $v / U$ and velocity is scaled with $U$. Figure 2 shows computed contour plots of the normalized velocity magnitude $|\boldsymbol{u}| / U$, with space scaled as $(x U / \nu, y U / v)$, for a wide range of non-dimensional times, $\tau \in\left[300,5 \times 10^{4}\right]$, 
(a) $t U / H=1\left(\tau=10^{4}\right)$

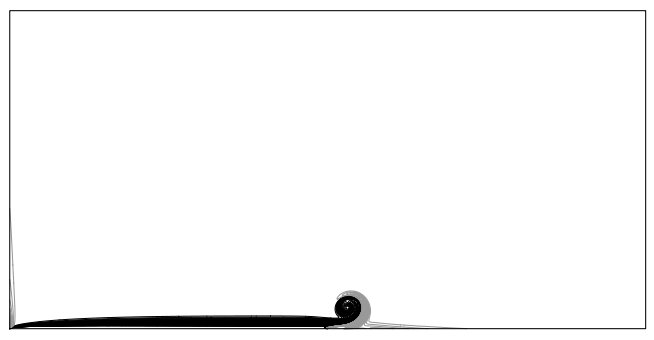

(c) $t U / H=3\left(\tau=3 \times 10^{4}\right)$

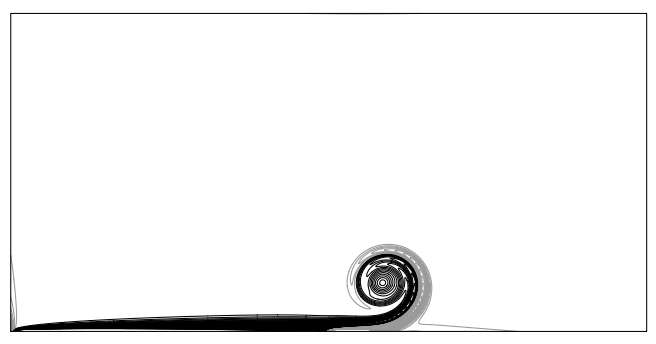

(e) $t U / H=5\left(\tau=5 \times 10^{4}\right)$

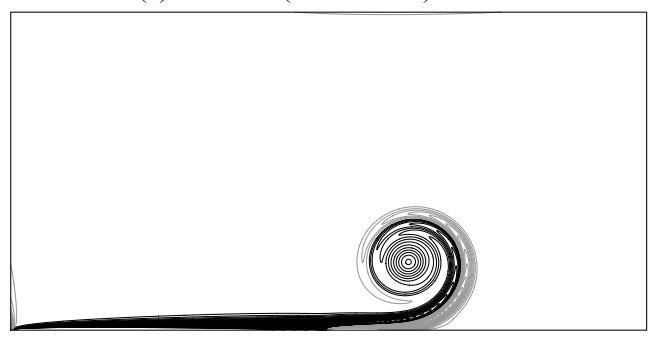

(b) $t U / H=2\left(\tau=2 \times 10^{4}\right)$

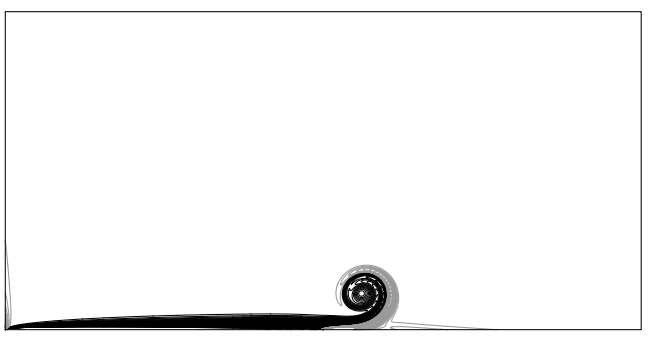

(d) $t U / H=4\left(\tau=4 \times 10^{4}\right)$

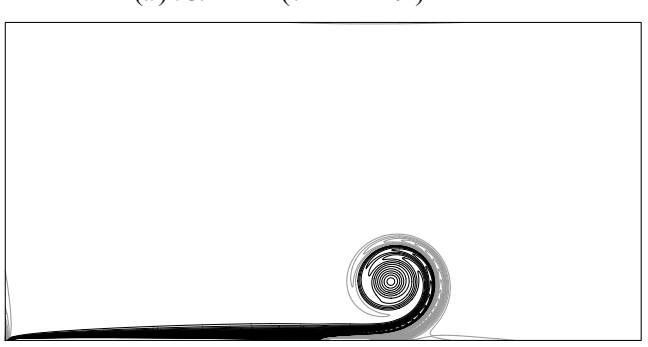

(f) $t U / H=10\left(\tau=10^{5}\right)$

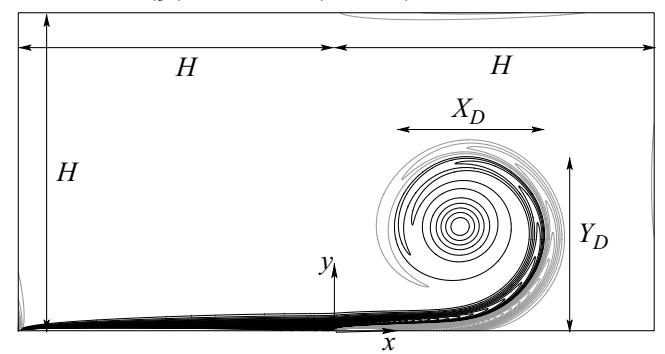

FIGURE 1. Vorticity contours following an impulsive start of the half-floor at $t=0$, at times as indicated, for $R e=10^{4}$. There are 50 positive contours (black) and 50 negative contours (grey) over the range $[-50,50]$; the vorticity range in the domain is $[-1180,1610]$ but the extreme values are in the boundary layer on the floor.

corresponding to simulations with $R e$ varying from 500 to $8 \times 10^{4}$ at various times (note that $t U / H=\tau / R e$ ). The data we have used to produce these plots correspond to times when the vortex structure is at least an order of magnitude smaller than the computational domain. It is clear that the scaled velocity fields at a given $\tau$ are qualitatively similar over a large range of $R e$ during the evolution while the size of the roller is small compared to $H$. There are some small quantitative differences primarily due to confinement effects. At a given $\tau$, the size of the roller compared to $H$ is smaller for larger $R e$. The size and shape of the roller structure in terms of $(x U / v, y U / v)$ is primarily a function of the control parameter $\tau$. For $\tau=300$, the structure appears as a vorticity front. For $\tau=4 \times 10^{3}$, it appears to have developed a rotational core, while for $\tau=5 \times 10^{4}$, the Stokes layer from the moving wall can be seen clearly wrapping around the large rotational structure. In the following sections, we examine the nature of the developing self-similar form. 
(a)
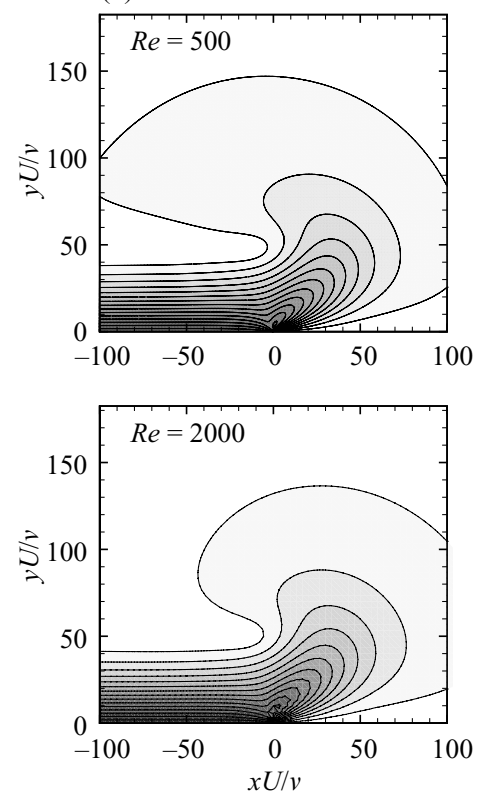

(b)
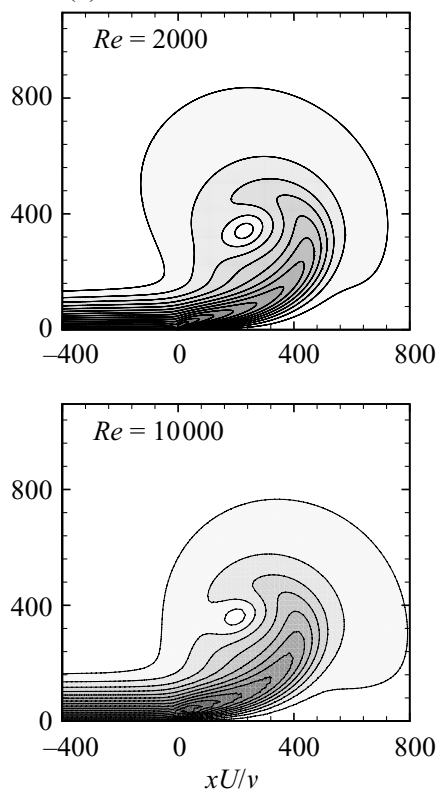

(c)
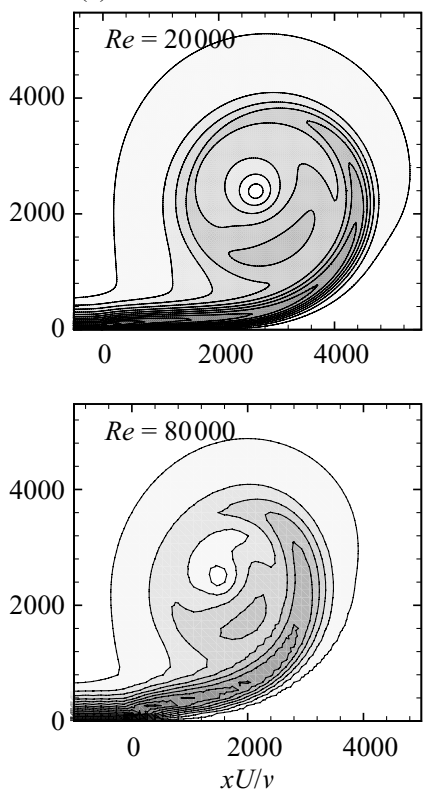

Figure 2. Contours of the normalized velocity magnitude $|\boldsymbol{u}| / U$ for $(a) \tau=300,(b) \tau=4 \times 10^{3}$ and $(c) \tau=5 \times 10^{4}$, over a range of $R e$ as indicated. There are 20 uniformly spaced contour levels between 0 and 1 ; white corresponds to 0 and black to 1 .
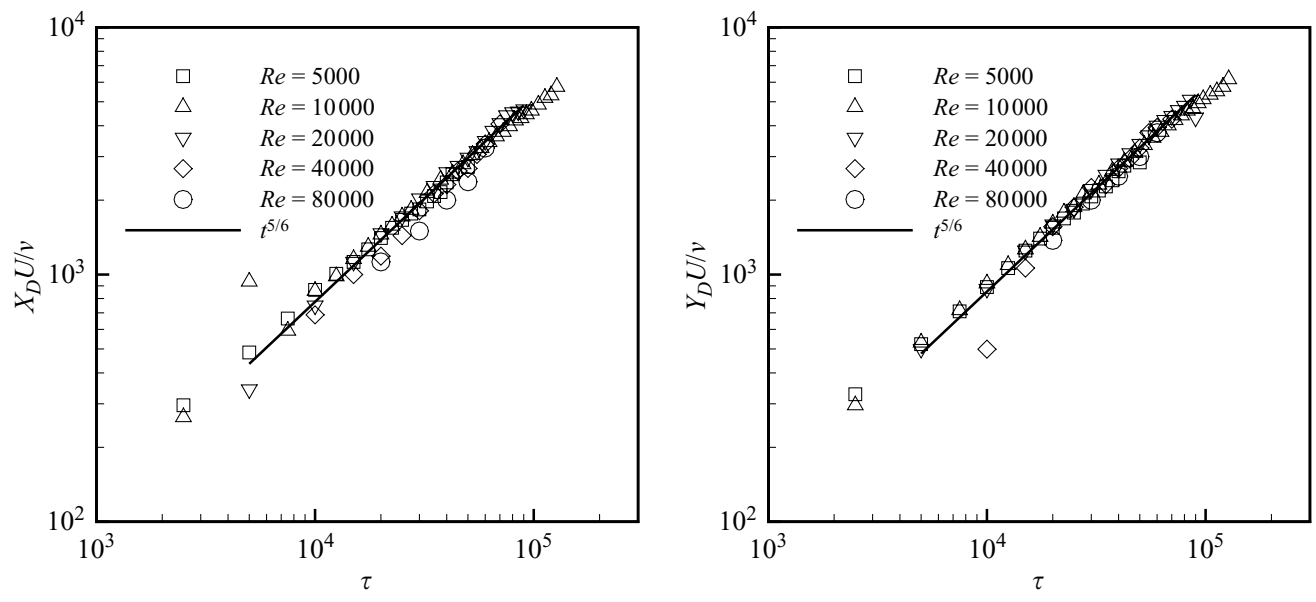

Figure 3. (a) $X_{D}$ vortex diameter data and $(b) Y_{D}$ vortex diameter data.

\subsection{Inertial scaling}

For $\tau>10^{3}$, a vortex roller can be clearly identified, and we characterize it, as was done in Allen \& Naitoh (2007), by its diameter in the $x$-direction, $X_{D}$, and its vertical extent above the floor, $Y_{D}$, as identified in figure $1(f)$. The variation of $X_{D}$ and $Y_{D}$, scaled by $v / U$, during the flow evolution from $\tau \approx 10^{3}$ to $\tau \approx 10^{5}$, for several cases with $R e$ ranging from $5 \times 10^{3}$ to $8 \times 10^{4}$, are shown in figure 3 . All the data collapse quite well onto a single curve which varies as $t^{5 / 6}$. This is the same inertial scaling that was observed experimentally for the self-similar development of the vortex roller in Allen \& 

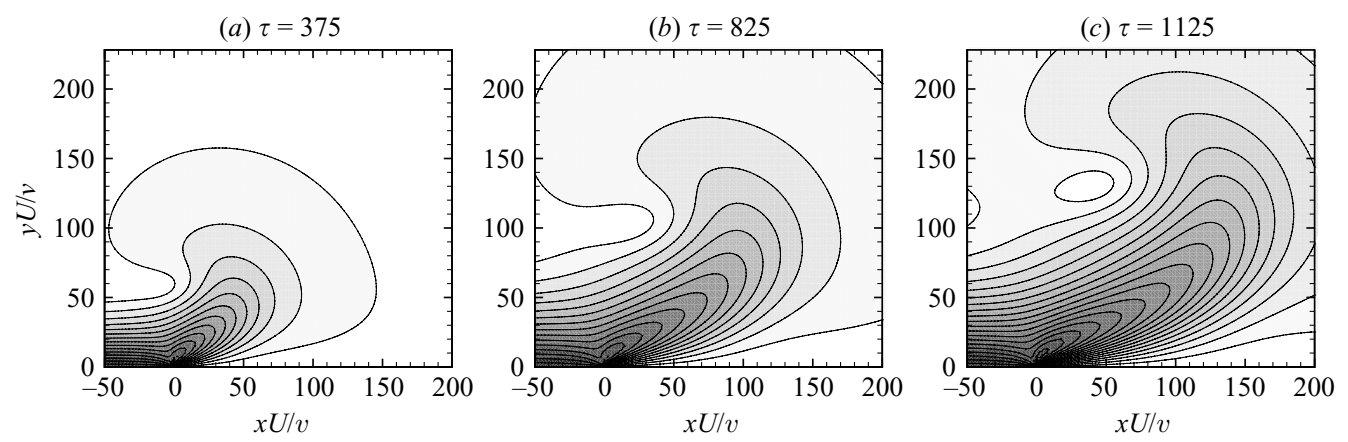

FIGURE 4. Contours of the normalized velocity magnitude $|\boldsymbol{u}| / U$ for $R e=1500$ at viscous times $\tau$ as indicated.

Naitoh (2007). Self-similarity breaks down when the size of the structure approaches the domain size in both experiments and computations. In the experiments, the vortex roller can become unstable to three-dimensional spanwise instabilities at early $\tau$ before the roller dimensions are comparable to the domain size. This is due to various effects including interactions between the roller and the spanwise endwalls of the flow apparatus as well as inherent three-dimensional instabilities of the roller which are analogous to the three-dimensional instabilities of the rollers in the wakes of bluff bodies. As a result, the two-dimensional simulations of the junction vortex flow have an extended self-similar range in $\tau$ compared to the experiments.

Simulation data allow us to probe the early $\tau$ development of the vortex roller. Figure 4 shows a sequence of the normalized velocity magnitude $|\boldsymbol{u}| / U$ for $\tau \in$ $[375,1125]$. Over this range of $\tau$ there is a significant change in the form of the velocity field. For $\tau<10^{3}$ the velocity magnitude contours have the form of a front without a clear local minimum. The development of an inflection point behind the leading vortex front can be seen in figure 4(b), and in figure 4(c) the appearance of what appears to be a vortex core (located at about $x U / v=40$ and $y U / v=130$ in figure $4 c$ ). The appearance of the core is important as it provides a point which can be tracked and used as a length scale to describe the developing structure.

The $t^{5 / 6}$ scaling obtained from the $X_{D}$ and $Y_{D}$ data suggests that inertial forces are dominant in the evolution of the vortex roller for $\tau>10^{3}$. For $\tau<10^{3}$, however, neither the simulations nor the experimental data provide a marker point that can be identified as a vortex core. At very early times following the impulsive start of the floor motion the flow must be dominated by viscous forces before transitioning to the inertially dominated flow regime. In the following sections, we analyse the numerical data for $\tau<10^{3}$ to explore the viscous-dominated early evolution of the flow.

\subsection{Viscous scaling}

During the initial formation of the roller structure, one expects viscous forces to dominate and for the spatial scales to develop proportional to $\sqrt{v t}$. Figure 5 shows contour plots of the velocity magnitude, $|\boldsymbol{u}| / U$, scaled spatially as $\xi=x / \sqrt{v t}, \eta=y / \sqrt{v t}$ for non-dimensional times of $\tau=1.5,17.5$ and 125. For over two orders of magnitude in $\tau$, it appears that the roller structure is developing on a viscous scale, as the plots of velocity magnitude appear almost identical.

In the study of viscous starting jets in the absence of apparatus length scales, Cantwell (1986) showed that the Stokes equations admit similarity transforms of the 

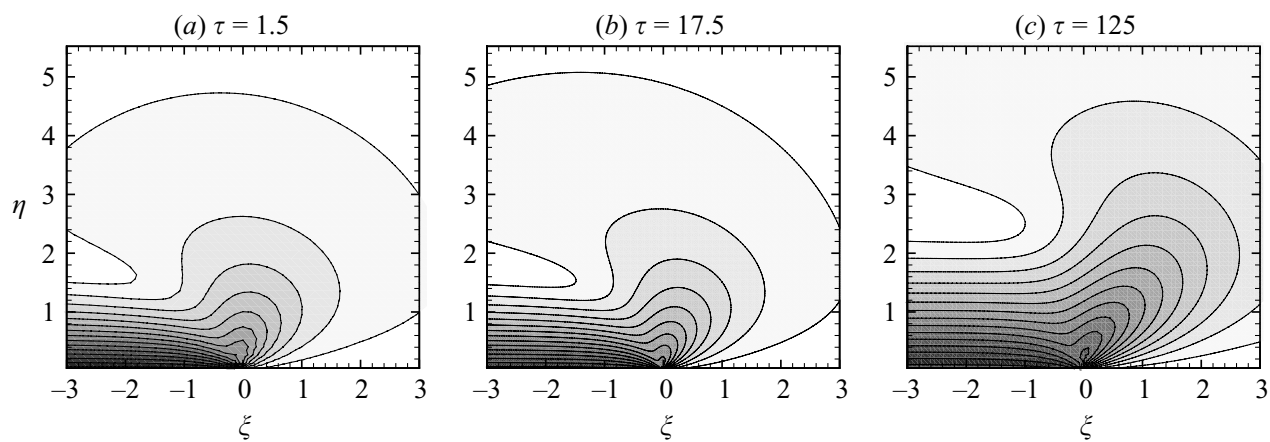

FIGURE 5. Contours of the normalized velocity magnitude $|\boldsymbol{u}| / U$ plotted in the viscous-scaled coordinates $\xi$ and $\eta$, for $(a) \tau=1.5,(b) \tau=17.5$ and $(c) \tau=125$. Contour levels in all frames are the same, with 10 contour levels in the range $|\boldsymbol{u}| / U \in[0,1]$.

form

$$
\xi=\frac{x}{\sqrt{v t}}, \quad \eta=\frac{y}{\sqrt{v t}}, \quad W=\frac{4 v(n-1) u}{M t^{n-2}}, \quad V=\frac{4 v(n-1) v}{M t^{n-2}} .
$$

The index $n$ is related to the nature of the forcing of the viscous starting jet. The derivation relied on the requirement that this impulse remain invariant under transformation. The constant $M$ is related to the impulse delivered to the flow.

In our junction vortex flow problem, there is no obvious invariant that is to be rendered constant. However, the scaled numerical results presented in figure 5 suggest that the appropriate velocity scaling is $|\boldsymbol{u}| / U$ and that the spatial scaling is $x / \sqrt{v t}$. Although the action of the moving wall is to apply a distributed force along its length and Cantwell's force was a point force applied at the origin of an unbounded domain, the above scalings suggest an analogy between Cantwell's solutions and our problem in the immediate neighbourhood of the junction between the moving and stationary bottom. The evolving flow topology to be described in $\S 3.3$ also follows a similar evolution to that described in Cantwell (1986) and hence the action of the velocity discontinuity in the immediate junction region may be considered akin to the step function forcing of Cantwell (1986).

\subsection{Viscous entrainment diagrams}

A problem with the interpretation of unsteady streamline patterns and velocity magnitude plots is that critical features are a function of the velocity of the observer. As explained in Cantwell (1986), a technique that removes this ambiguity with unsteady flow fields is the use of entrainment diagrams which show particle paths. For an unsteady flow field, particle paths would typically cross, however, by using similarity transforms the potential exists to develop universal entrainment diagrams for unsteady flows. The equations for particle paths are

$$
x_{t}=u, \quad y_{t}=v .
$$

Using the viscous similarity scalings for space and velocity given in (3.1), these particle path equations are transformed into

$$
\xi_{\alpha}=\sqrt{\tau} W-0.5 \xi=F, \quad \eta_{\alpha}=\sqrt{\tau} V-0.5 \eta=G,
$$

where $\alpha=\ln (t)$. The origin of the $(\xi, \eta)$ coordinate system is the junction of the moving wall with the stationary one. From our computational data and equation (3.3), the local values of $\xi_{\alpha}$ and $\eta_{\alpha}$ are calculated. The technique of isolines can then be used to 
produce an entrainment diagram in the similarity coordinates $(\xi, \eta)$. The scaled time $\tau$ plays the role of a control parameter in this system.

This numerical empirical approach to the evolving topology of the entrainment diagram shows the viscous flow is dominated by critical points that are created and change their characteristics as $\tau$ increases. Critical points in the self-similar entrainment diagram are points where the slope of $\mathrm{d} \xi / \mathrm{d} \eta$ is indeterminate. These points are found by solving $F=0$ and $G=0$ simultaneously. Near the critical point the flow field is governed by

$$
\left[\begin{array}{l}
\xi_{\alpha} \\
\eta_{\alpha}
\end{array}\right]=\left[\begin{array}{cc}
F_{\xi} & F_{\eta} \\
G_{\xi} & G_{\eta}
\end{array}\right]\left[\begin{array}{l}
\xi-\xi^{\star} \\
\eta-\eta^{\star}
\end{array}\right],
$$

where $\left(\xi^{\star}, \eta^{\star}\right)$ is the critical point and the Jacobian matrix is evaluated at the critical point. The nature of the critical points is then defined by the trace and determinant of the Jacobian matrix, as outlined in Perry \& Chong (1987).

Figure 6 shows the viscous entrainment diagrams for a range of $\tau$, from 0.5 to 100 . For very small $\tau$, there is a single stable node point very near the junction on the floor. As $\tau$ increases, this node migrates in the positive $x$ direction and the trajectories approaching it rapidly converge on to a sharp front before slowly approaching the node (figure $6 a-d$ ). As $\tau$ increases beyond $\tau=40$, a saddle-node point develops on this front, which bifurcates to form a saddle point and a stable node point on the front (figure 6e). The flow in toward the front is fast and the subsequent flow along the front is slow. The fast/slow nature of the flow in the neighbourhood of the front is characterized by the magnitude of the eigenvalues of the Jacobian matrix in (3.4) evaluated at the saddle point. For example, at $\tau=45$ (figure $6 e$ ), the negative eigenvalue of the saddle point is -1.04 (the corresponding eigenvector is $(1.0,-0.61)$, approximately normal to the front), whereas the positive eigenvalue is an order of magnitude smaller, 0.11 (the corresponding eigenvector is $(1.0,1.3)$, approximately tangential to the front). With further increases in $\tau$, the saddle point migrates along the front towards the node near the wall, and the other node which now terminates the front, evolves into a stable spiral node, signalling the roll-up of the vorticity front and the formation of a vortex core (figure $6 f$ ). The final topology of critical points produces a pattern that is the same as that proposed by Allen \& Naitoh (2007) for high $\tau$ flow based on experimental observations.

In this paper we have used the definition of the appearance of a spiral node in the entrainment diagram, which occurs at $\tau \simeq 50$, as the criterion for vortex formation. Physically, this is the time at which a dye visualization streakline would begin to rotate around itself. The reason for the large difference between the times for the appearance of a spiral node and the appearance of a local minimum in the velocity field is that the flow field is unsteady and hence streaklines and streamlines are not coincidental.

\subsection{Limits of the corner flow behaviour}

The near-field behaviour of the flow field is described by Taylor's creeping corner flow solution. In polar coordinates, Taylor (1960) suggested $\psi(r, \theta)=r f(\theta)$ is a solution to the streamfunction in the immediate vicinity of the moving wall/stationary wall junction and that the flow in this region is dominated by viscous forces. Substitution into the bi-harmonic equation $\nabla^{4} \psi=0$, or equivalently $\nabla^{2} \omega=0$, yields

$$
\frac{d^{4} f}{d \theta^{4}}+2 \frac{d^{2} f}{d \theta^{2}}+f(\theta)=0 .
$$



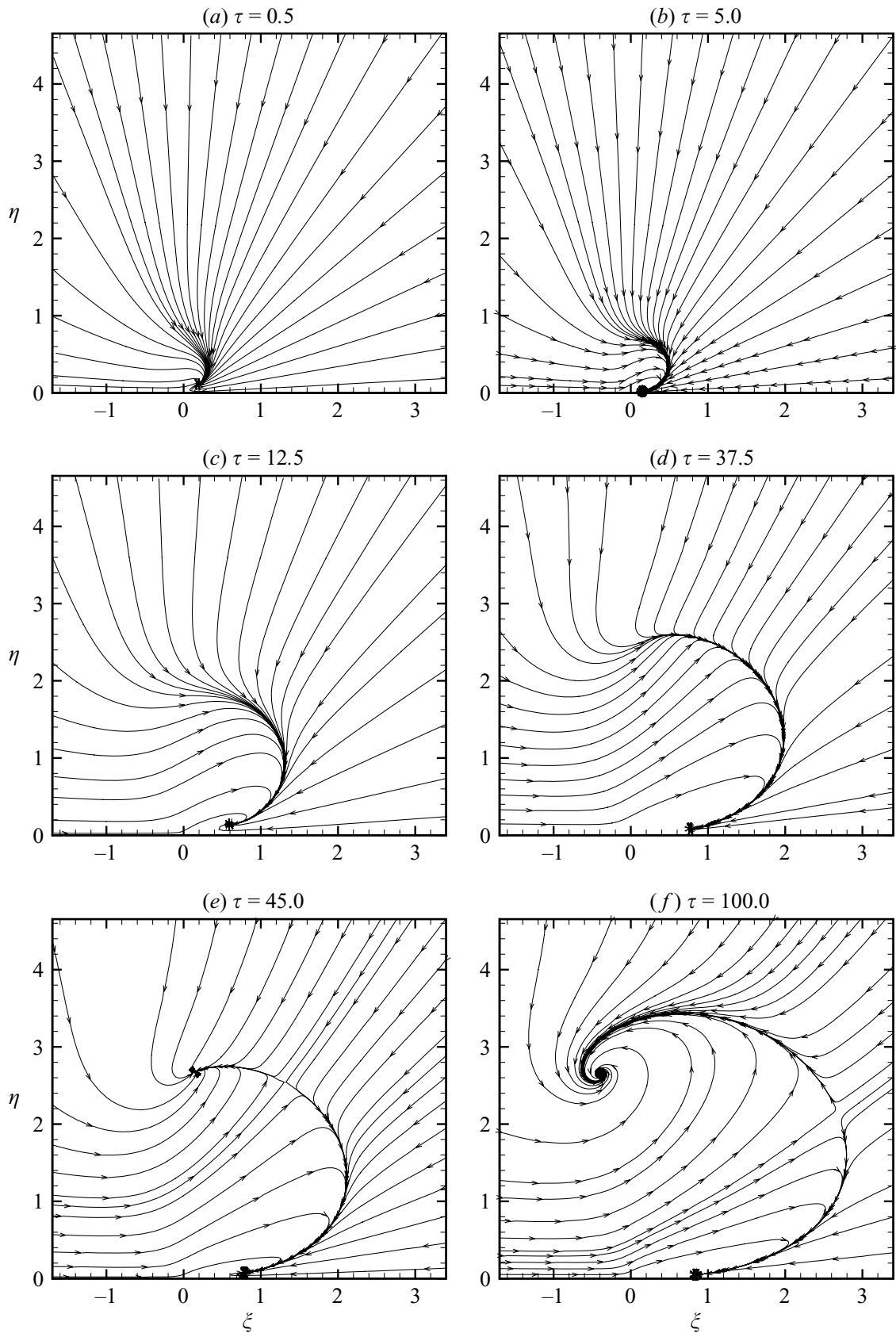

FIGURE 6. Self-similar entrainment diagrams for $\tau$ as indicated.

The solution for the streamfunction with appropriate boundary conditions is $\psi / \nu=r U \theta \sin (\theta) / v$ and a plot of the solution for the streamfunction $\psi(x, y) / v$ is shown in figure $7(a)$. The most striking aspect of this plot is the divergence of the streamlines away from the stationary surface. This solution gives no indication of the formation of a vortex roller. As a comparison, figure 7(b) shows stream traces from the simulations at $\operatorname{Re}=100, \tau=0.35$ for $r U / v<0.1$. The plot shows similar features 

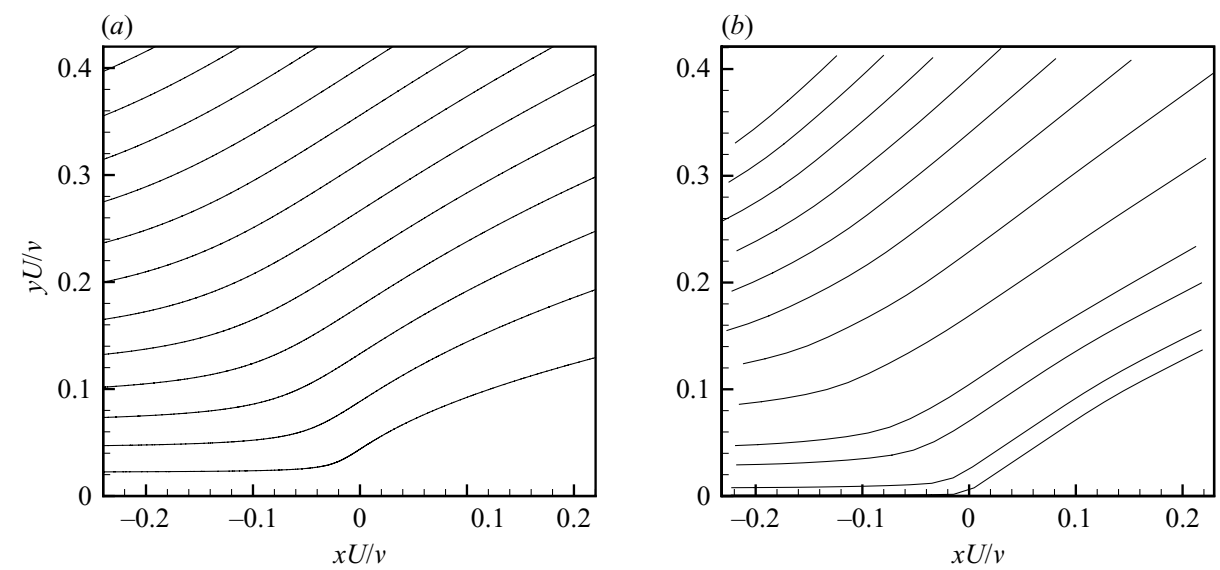

FIGURE 7. (a) Taylor's self-similar viscous solution and (b) comparison with computational data at small $r U / v<0.1$ at $\tau=0.35$.

to Taylor's solution indicating Taylor's solution dominates over any unsteady inertial effects in the region where viscous forces are large. As Taylor's solution is self-similar, an estimate of its range of validity comes from consideration of the relative size of the inertial terms described by this solution, when compared to the viscous ones. Taylor arrived at a practical criterion for the range of the viscous solution as being $r U / v \ll 1$, where $r$ represents the distance from the corner singularity.

\section{Conclusion}

Numerical simulations have been used to show that, in a geometry consisting of a moving wall sliding under a stationary one, a vortex roller is produced which exhibits self-similar growth if the roller is small compared to the computational domain. An appropriate non-dimensional time scale with which to describe the early vortex development is $\tau=t U^{2} / \nu$. Data from simulations for a wide range of Reynolds numbers, based on the characteristic size of the computational domain and velocity of the moving wall, were collapsed when scaled with respect to $\tau$. Simulations for $\tau>10^{3}$, in the inertial regime of vortex development, are in good agreement with the experimentally observed $t^{5 / 6}$ scaling of Allen \& Naitoh (2007).

A principal result of this paper has been to classify the transition of the flow from viscous to one where the flow is dominated by inertial forces, and to identify when the vortex forms. Simulation data show that, for $\tau<100$, the flow field scales in a self-similar fashion with a viscous time scale, $\sqrt{t}$. The simulations show that the flow field topology undergoes a transition from a non-rotating vorticity front to a rotating vortex at $\tau \simeq 50$. This result was determined with the use of entrainment diagrams in self-similar coordinates, which were used to show that the initial flow field resembles a vorticity front with a single node on the $x$-axis with the trajectories leading in toward it first focusing in on a slow manifold which we identify as the front. Along this slow manifold at about $\tau=40$, a saddle-node point develops that subsequently splits into a saddle point and a stable focus. The unstable manifolds of the saddle are the slow directions and these flow into the two stable nodes, the original one on the $x$-axis and the newly formed one in the flow interior. This interior node transforms into a spiral focus at $\tau \simeq 50$. It is the appearance of the spiral focus that constitutes the formation of a rotational vortex at the head of the vorticity front. This early evolution develops 
on the viscous time scale $\sqrt{t}$. This is then followed by a transitional regime, and by $\tau \sim 10^{3}$ the flow develops on an inertial time scale $t^{5 / 6}$. Throughout this whole evolution, the flow field in a neighbourhood of the junction between the stationary and moving wall is well described by Taylor's self-similar viscous solution.

It would be of interest to investigate other flows that are driven by jump discontinuities in the velocity boundary conditions to see if analogous self-similar scalings describe their local behaviour.

\section{REFERENCES}

Allen, J. J. \& Chong, M. S. 2000 Vortex formation in front of a piston moving through a cylinder. J. Fluid Mech. 416, 1-28.

Allen, J. J. \& Naitoh, T. 2007 Scaling and instability of a junction vortex. J. Fluid Mech. 574, 1-23. Cantwell, B. J. 1986 Viscous starting jets. J. Fluid Mech. 173, 159-189.

Conlon, B. P. \& Lichter, S. 1995 Dipole formation in the transient planar wall jet. Phys. Fluids 7, 999-1014.

Guezet, J. \& Kageyama, T. 1997 Aerodynamic study in a rapid compression machine. Revue Generale de Thermique 36, 17-25.

Hughes, M. D. \& Gerrard, J. H. 1971 The stability of unsteady axisymmetric incompressible pipe flow close to a piston. Part 2. Experimental investigation and comparison with computation. J. Fluid Mech. 50, 645-655.

Koseff, J. R. \& Street, R. L. 1984a The lid-driven cavity flow: A synthesis of qualitative and quantitative observations. J. Fluids Engng. 106, 390-398.

Koseff, J. R. \& Street, R. L. 1984b Visualization studies of a shear driven 3-dimensional recirculating flow. J. Fluids Engng. 106, 21-29.

Lopez, J. M. \& SHEN, J. 1998 An efficient spectral-projection method for the Navier-Stokes equations in cylindrical geometries. Part I. Axisymmetric cases. J. Comput. Phys. 139, 308-326.

Perry, A. E. \& Chong, M. S. 1987 A description of eddying motions and flow patterns using critical-point concepts. Annu. Rev. Fluid Mech. 19, 125-155.

TabaczYnSKi, R. J., Hoult, D. P. \& KeCK, J. C. 1970 High Reynolds number flow in a moving corner. J. Fluid Mech. 42, 249-255.

TAYLOR, G. I. 1960 Aeronautics and Aeromechanics. Pergamon. 